\title{
Study of Path Planning Algorithm Based on Fuzzy Logic
}

\author{
Dianhua Zhang \\ School of Computer Engineering and Science \\ College of Digital Arts, \\ Shanghai University \\ Shanghai, China \\ bandit05@163.com \\ Yimin Chen * \\ * Corresponding author \\ School of Computer Engineering and Science \\ Shanghai University \\ Shanghai, China \\ bandit05@sina.com
}

\author{
Chen Huang \\ School of Computer Engineering and Science \\ Shanghai University \\ Shanghai, China \\ channinghuang@shu.edu.cn
}

\author{
Mingke Gao \\ School of Computer Engineering and Science \\ Shanghai University \\ Shanghai, China \\ gaomingke@shu.edu.cn
}

\begin{abstract}
The path planning problem is an important problem in the research area of robot, games and group animation. However, the current path planning algorithms don't consider practical problems such as roughness, gradient, and barrier height etc. This paper shows a 2.5dimensional terrain grid which can reduce the amount of computation. By applying the fuzzy logic theory, the terrain trafficability of the rugged road can be evaluated based on different gradient, roughness, elevation difference; the trafficability factor can be achieved and applied to the heuristic function. The improved algorithm can solve the symmetry problem of path planning on uneven surfaces, reduce the search space, and improve the search speed.
\end{abstract}

Keywords-path planning; fuzzy logic; trafficability factor; terrain grid; rugged road

\section{INTRODUCTION}

Path planning, which has a wide range of applications in mobile robotics, three-dimensional games and other fields, is to find an optimal path from the starting point to the target point in the state space based on certain criteria. The research can be divided into global path planning, local path planning, hybrid path planning. Global path planning includes the $\mathrm{A} *$ algorithm, Dijkstra graph algorithm, visibility graph method, tangent diagram method, topological methods. Local path planning refers to artificial potential field method [1-2], fuzzy logic method [3], D* algorithm, neural networks, genetic algorithms, ant colony algorithm.

The concept of fuzzy set theory is put forward by the control expert L.A.Zadeh in 1965. By combining the appropriate expertise with fuzzy logic, using the similar method of natural language, the fuzzy set theory can achieve a good effect when it deals with uncertain data. It has a strong ability to overcome the noise and uncertainty.

Nowadays, the research of $\mathrm{A}^{*}$ has aimed not only to find the shortest path, but to find ways to reduce its search space and improve search speed. However, the path finding algorithms often encounter the problem of symmetry, that means although only one path is needed, all the paths with the same f values will be searched, which would greatly reduce the efficiency of the search. At present, many algorithms to solve the similar problems exist.

In RSR method (Rectangular Symmetry Reduction Method), grids are merged into rectangular grids without internal barrier regions. Nodes in the rectangular area are deleted [4,5]. If the beginning or end node is in the rectangular, the insert operation is performed to subdivide the rectangular area. JPS (Jump Point Search) algorithm is based on the $\mathrm{A} *$ algorithm to accelerate the search process, which can solve the problem of symmetry $[6,7]$.

This paper is to study the path planning of the virtual vehicle on the rugged road created by $2.5 \mathrm{D}$ grids. Fuzzy logic theory is used to elevate the terrain trafficability of the rugged road with different gradient, roughness, elevation difference. The passable factor can be got and applied to the heuristic function. Improved algorithm solves the symmetry problem of planning on uneven surfaces created by $2.5 \mathrm{D}$ grids, which reduces the search space and speeds up the search speed.

\section{TERRAIN DATA PROCESSING}

Firstly, buildings, tall trees, rivers etc. are marked as the unavailable by using boolean grids. For the available area, the gradient, roughness, barrier height are chosen to go through the quantitative research; and then the fuzzy logic is used to the qualitative analysis.

\section{A. The analysis of terrain}

Chinese standard road slope angle generally does not exceed $30^{\circ}$. For cars driving in cities or on highway, the maximum slope angle is about $10^{\circ}$. Since trucks often drive on bad roads, the maximum slope angle that trucks can pass through should be about $16.5^{\circ}$. 
The initial maximum obstacle height of virtual vehicle is defined as $h_{\max }=40 \mathrm{~cm}$. For easy discussion, the initial slope angle can be taken by a slightly larger range of values, viz., $a \in\left[0^{\circ}, 45^{\circ}\right] \cup\left[-180^{\circ},-120^{\circ}\right]$. Before path planning, the three-dimensional terrain is preprocessed according to the slope angle. The terrain that the vehicle can't pass through is marked impassable as follows:

1) Slope angle $\alpha \in\left[0^{\circ}, 45^{\circ}\right] \cup\left[-180^{\circ},-120^{\circ}\right]$, this is the slope angle that the vehicle can pass through. When $0^{\circ}<\alpha \leq 45^{\circ}$, it is the positive gradient that the vehicle can pass through. When $-180^{\circ}<\alpha \leq-120^{\circ}$, it is the negative slope angle that the vehicle can pass through.

2) When $\alpha \in\left(45^{\circ}, 180^{\circ}\right)$, the gradient is defined as vertical plane.

3) When $\alpha \in\left(-120^{\circ}, 0^{\circ}\right)$, the plane is described as cliffs or deep groove. The corresponding grid point is also marked as not impassable.

\section{B. The calculation of gradient}

Gradient is a vector, its mold should be equal to the tangent of the angle of tangent plane and the horizontal plane. Its direction is equal to the projection direction in the horizontal plane of the largest tilt direction vector on the tangent plane(namely aspect). The gradient of any surface is equal to the vector sum of gradients in vertical direction on the plane. The gradient can be calculated by the following equation[8]:

$$
\tan \alpha=\sqrt{g_{x}^{2}+g_{y}^{2}}
$$

$g_{x}$ and $^{g_{y}}$ is the change rate of elevation in the $\mathrm{x}$ and $\mathrm{y}$ direction, $\alpha$ is the slope angle. In order to calculate $g_{x}$ and ${ }^{g_{y}}$, it is necessary to fit surface, which requires solving the equations. Since the computation is complexity, the approximate formula may be used as follows:

$$
\tan \alpha=\sqrt{(\Delta z / \Delta x)^{2}+(\Delta z / \Delta x)^{2}}
$$

$h_{a} 、 h_{b} 、 h_{c} 、 h_{d}$ is the vertex elevation of square grid.

$l$ is the side length of grid. The gradient can be calculated as follows: $\tan \alpha=\sqrt{u^{2}+v^{2}}, u=\sqrt{2}\left(h_{a}-h_{c}\right) / 21$, $V=\sqrt{2}\left(h_{d}-h_{b}\right) / 21$. Xuejun Liu finds that the calculation method of gradient using the adjacent four grids has the highest grade accuracy and efficiency, followed by Horn algorithm [9].

\section{Surface roughness}

Roughness is used to indicate the undulating ground. For a square grid, the roughness can be expressed by the midpoint distance ${ }^{R_{d}}$ of the diagonal line L1 and L2 of grid vertices, as is shown in Fig.1.

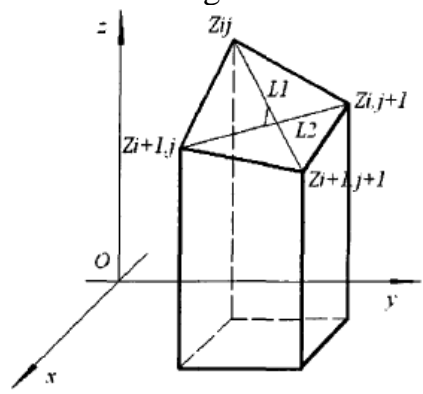

Figure 1. The calculation of roughness
$\mathrm{R}_{\mathrm{d}}=\left|\left(z_{i+1, j+1}-z_{i, j}\right) / 2-\left(z_{i, j+1}-z_{i+1, j}\right) / 2\right|$

$=\frac{1}{2}\left|z_{i+1, j+1}-z_{i, j}-z_{i, j+1}+z_{i+1, j}\right|$

Roughness $\mathrm{R}=\mathrm{R}_{\mathrm{d}} / \mathrm{R}_{\max }, \mathrm{R}_{\max }$ represents the maximum distance between the midpoint of all the grid, so that the roughness is limited to $[0,1]$. Larger values indicate greater roughness, whereas the flat ground.

\section{Obstacle height}

The size of grid is defined by the size of the projection area of the virtual vehicle. In order to show the uneven pavement in the 2.5-dimensional grid, the resolution need to be improved. The height of grid is an average value of all grid heights in the projection area of the virtual vehicle.

$$
h=\frac{1}{n} \sum_{i=1}^{n} z_{i}
$$

The height difference of the grid where the virtual vehicle is located in and the adjacent grid can be got from the average height difference, just as follows.

$$
\Delta h=\left|h_{1}-h_{2}\right|
$$

\section{TRAFFIC ABILITY}

The theory of fuzzy logic is used to analyze the trafficability, the reasoning process is shown in Fig.2:

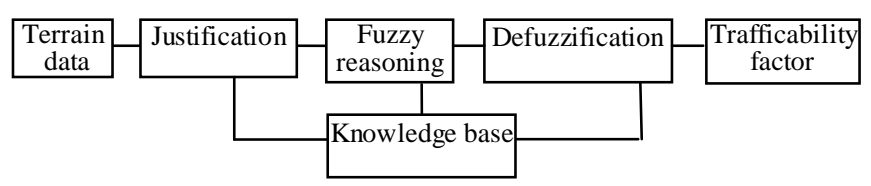

Figure 2. The reasoning process of fuzzy logic

\section{A. Fuzzification}

Fuzzification becomes the input data to fuzzy data. Input data are mainly gradient, roughness and the barrier height. Firstly, the input data is processed and included in the scope of their domain. The fuzzy data will be represented by fuzzy sets.

The triangular membership functions are used when the data is in fuzzy processing:

$$
f(x: a, b, c)=\left\{\begin{array}{cc}
0 & x \leq a \\
\frac{x-a}{b-a}, & a \leq x \leq b \\
\frac{c-x}{c-b}, & b \leq x \leq c \\
0 & c \leq x
\end{array}\right\}
$$

The following discussion is about uphill:

When $\tan \alpha \in[0,0.087)\left(\alpha \in\left[0^{\circ}, 5^{\circ}\right)\right.$ ), the situation is defined as easy. When $\tan \alpha \in[0.087,0.2679)$ ( $\alpha \in\left[5^{\circ}, 15^{\circ}\right)$ ), the situation is defined as normal. When $\tan \alpha \in[0.2679,0.4663)\left(\alpha \in\left[15^{\circ}, 25^{\circ}\right)\right.$, the situation is defined as fairly normal. When $\tan \alpha \in[0.4663,1]$ ( $\alpha \in\left[25^{\circ}, 45^{\circ}\right]$ ), the situation is defined as hard.

When the roughness $R \in[0,0.4)$, it is described as smooth. When the roughness $R \in[0.4,0.7)$, it is described as fairly rough. When the roughness $R \in[0.7,1]$, it is described as rough. 
When the obstacle height $\Delta h \in[0,0.2)$, it is described as being easy. When the obstacle height $\Delta h \in[0.2,0.4)$, it is described as being difficult.

Trafficability is expressed as \{low, medium, high $\}$. Low means that it could not go through. Medium indicates the possibility of a medium through. High expresses that the virtual vehicle can go through. The higher the value is, the higher the reachability is.

\section{B. Knowledge base}

Knowledge base is a series of fuzzy control rules described by fuzzy language. It reflects the expert experience. Fuzzy rules are defined in Table 1:

TABLE I. THE FUZZY RULES

\begin{tabular}{|l|l|l|l|}
\hline $\begin{array}{l}\text { obstacle } \\
\text { height }\end{array}$ & slope & roughness & $\begin{array}{l}\text { Traffica } \\
\text { bility }\end{array}$ \\
\hline easy & easy & smooth & high \\
\hline easy & normal & smooth & high \\
\hline easy & fairly normal & smooth & medium \\
\hline easy & hard & smooth & medium \\
\hline easy & easy & fairly rough & medium \\
\hline easy & normal & fairly rough & medium \\
\hline easy & fairly normal & fairly rough & low \\
\hline easy & hard & fairly rough & low \\
\hline$*$ & $*$ & rough & low \\
\hline difficult & $*$ & $*$ & low \\
\hline$*$ & hard & $*$ & low \\
\hline difficult & easy & smooth & medium \\
\hline difficult & normal & smooth & medium \\
\hline difficult & fairly normal & smooth & low \\
\hline difficult & hard & smooth & low \\
\hline difficult & easy & fairly rough & medium \\
\hline difficult & normal & fairly rough & medium \\
\hline difficult & fairly normal & fairly rough & low \\
\hline difficult & hard & fairly rough & low \\
\hline
\end{tabular}

* Indicates that the value does not affect the result.

\section{Defuzzification}

Defuzzification is that the data represented by fuzzy language can be expressed as the trafficability factor. The trafficability factor $\mathrm{u}$ is got by using gravity method:

$$
u=\frac{\int V \bullet \mu_{v}(v) d V}{\int \mu_{v}(v) d V}
$$

$\mu, v$ refers to the fuzzy output and input, respectively.

\section{Experiment results of trafficability}

The result of trafficability is shown in Fig.3.

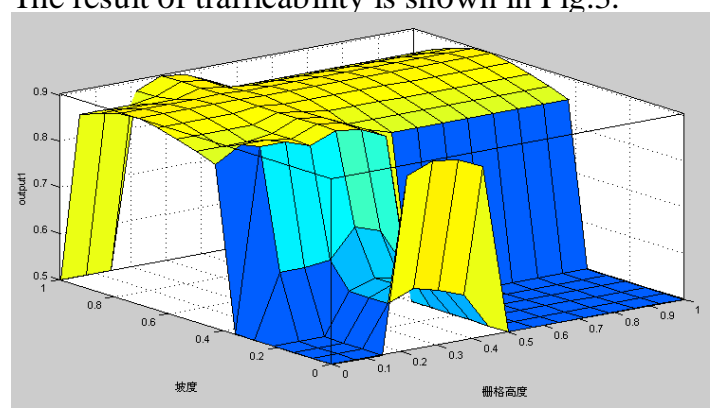

Figure 3. The result of trafficability

\section{MAP}

The representation of map includes triangular grid method and grid method. Grid method is divided into three-dimensional grid and two-dimensional grid. Data got by three-dimensional grid is redundant, the amount of data is big, which will affect the efficiency of the algorithm.

Boolean lattice is used more often in two-dimensional grid. Movement in grid is generally divided by plane motion, movement by side, vertex motion. Plane motion is prone to the jagged, so smooth operation is needed. Movement by side is mainly applied to the larger grid. 2.5 dimensional square grids are used to create the ground in the algorithm. The object moves along the movement direction of eight vertices.

2.5-dimensional grid is based on a two-dimensional grid, an increase in elevation. The average height of surface is recorded by each plane grid. An array of $h[x, y]$ represents elevation, $\mathrm{x}, \mathrm{y}$ refers to the number of the grid in the $\mathrm{X}$-axis and $\mathrm{Y}$-axis direction, so the plane is discrete. The terrain can be represented by finite dimensional vector sequence in the movement space of the model(MR).

$$
\left\{S_{i}=\left(x_{i}, y_{i}, h_{i}\right), i=1,2, \ldots, n\right\},\left(x_{i}, y_{i}, h_{i}\right) \in M R
$$

Storage space occupied by such terrain represented is as large as two-dimensional grid, but the elevation can be expressed as three dimensional grid map. Fong uses 2.5dimensional grids to create the terrain [10], Gutmann also uses 2.5 dimensional grids to represent the terrain containing obstacles [11].

2.5-dimensional grid map is obtained by connecting adjacent nodes. If there are not adjacent nodes, a quadrilateral is used to represent the grid. If there are adjacent nodes, two triangles are used to represent the grid. So a grid map is no longer stepped up. The surface of three dimensional grid needs $2,146,689$ storage units in this experiment, but the 2.5 dimensional grid requires only 16,641 storage units.

\section{IMPROVED JPS ALGORITHM}

\section{A. JPS algorithm}

JPS is evolved on the A * algorithm, and its heuristic function $f(x)=g(x)+h(x), g(x)$ is the cumulative cost from the initial point to the point $x, h(x)$ is the estimated cost from $x$ to the target point. Initially, JPS is released for the two-dimensional grid. Each node in grid has less than eight neighbors. The cost of translation is 1 . The cost of oblique movement is $\sqrt{2}$.JPS algorithm uses a pruning rule in the path search process. The rule is to trim impossible nodes by the direction from the parent node to the current point. The main idea is to cut off the neighbor nodes which can be reached from the parent nodes, so the symmetry problem can be solved. When expanding the adjacent points, not all the neighbors are placed into the open list. Only the points (forced neighbors) with obvious characteristics are expanded, such as inflection points and obstacle points. This greatly reduces the number of nodes in the open list. JPS algorithm does not require pretreatment and takes up very little memory. JPS algorithm can accelerate the $\mathrm{A} *$ algorithm several times 
and reduce the space occupied by $15-30$ times. The gray nodes surrounding obstacle grids represent the search space of the algorithm in Fig.4. As is shown in Fig.4, JPS algorithm can greatly reduce the search space, thereby reduce the useless search nodes, and increase the search speed.

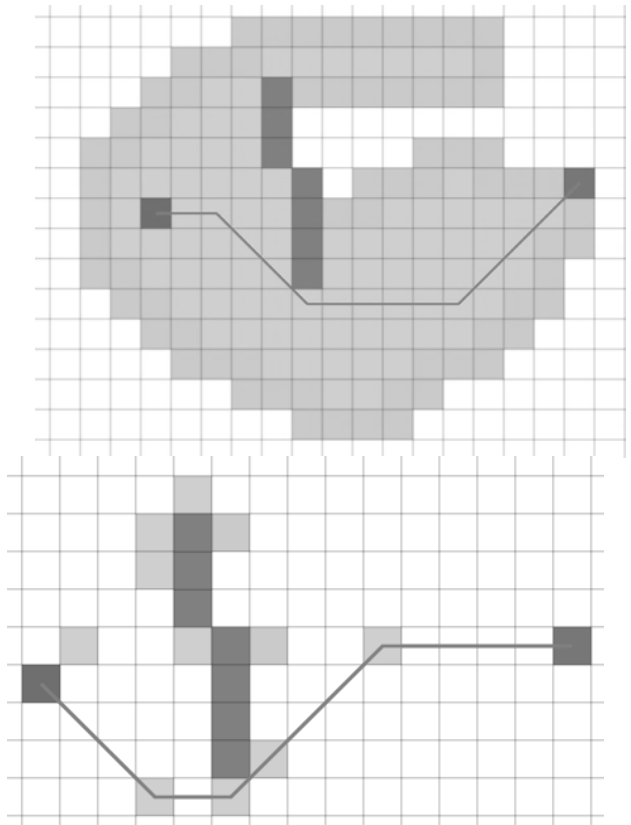

Figure 4. Ordinary A * algorithm and JPS algorithm

\section{B. 2.5D JPS algorithm}

\section{1) 2.5D diagonal distance}

Distances used in pathfinding algorithm on a grid map include Manhattan distance, diagonal distance, Euclidean distance. Different distances are used in JPS algorithm to compare efficiency. From Table 2, it can be seen that the algorithm using the diagonal length spends the least time. Time and iteration is expressed using integer.

TABLE II. COMPARE OF DIFFERENT DISTANCES

\begin{tabular}{|l|r|l|c|}
\hline Length & $\begin{array}{l}\text { Length } \\
\text { of path }\end{array}$ & Time & Iterations \\
\hline $\begin{array}{l}\text { Manhatta } \\
\text { n distance }\end{array}$ & 16.9 & $6 \mathrm{~ms}$ & 23 \\
\hline $\begin{array}{l}\text { Euclidean } \\
\text { distance }\end{array}$ & 16.9 & $10 \mathrm{~ms}$ & 22 \\
\hline $\begin{array}{l}\text { Diagonal } \\
\text { distance }\end{array}$ & 16.9 & $3 \mathrm{~ms}$ & 24 \\
\hline
\end{tabular}

The two-dimensional diagonal distance is extended to 2.5D, 2.5D diagonal distance is as follows: $\mathrm{D}(\mathrm{n})=\max (\mathrm{dx}, \mathrm{dy}, \mathrm{dh})+(\sqrt{2}-1)(1+(\sqrt{2}-1)) * \min (\mathrm{dx}, \mathrm{dy}, \mathrm{dh}) \quad$, $d x=\operatorname{abs}\left(\right.$ n. $\left.x^{-g} \cdot x\right), \quad d y=a b s\left(n \cdot y^{-g} \cdot y\right), \quad d h=a b s(n \cdot h-g . h)$ (8)

$\mathrm{n}$ is the coordinate of the moving object, $\mathrm{g}$ is the coordinate for the next point, dh refers to the elevation difference between different points. The diagonal distance between two points is approximately the length of the Euclidean distance, but with less complexity, with no frequent square and square root performance which reduce the complexity of the algorithm.

\section{2) Heuristic function design}

By using the distance only as heuristic function tend to ignore the real situation on the ground such as roughness, gradient, etc. The trafficability factor $u$ has been obtained by the fuzzy logic, so the heuristic function is designed as follows:

$$
\mathrm{h}(\mathrm{n})=\mathrm{D}(\mathrm{n})^{\mathrm{w}_{\mathrm{d}}} \frac{1}{\mathrm{u}^{\mathrm{w}_{\mathrm{u}}}}
$$

Where D (n) means the 2.5D distance from the initial node to the node $\mathrm{n}, \mathrm{u}$ is the trafficability factor, ${ }^{w_{d}}{ }^{w_{u}}$ represents the weight factor of distance and reachability.

\section{The effect of $2.5 D$ JPS algorithm}

The effect picture of 2.5D JPS algorithm on irregular path is shown in Fig.5. Path planning is from the world coordinates $(0,0,0)$ to $(250,0,250)$, with a time of $82.34787 \mathrm{~ms}$, path length 674 , the number of nodes used to generate the path 89 .

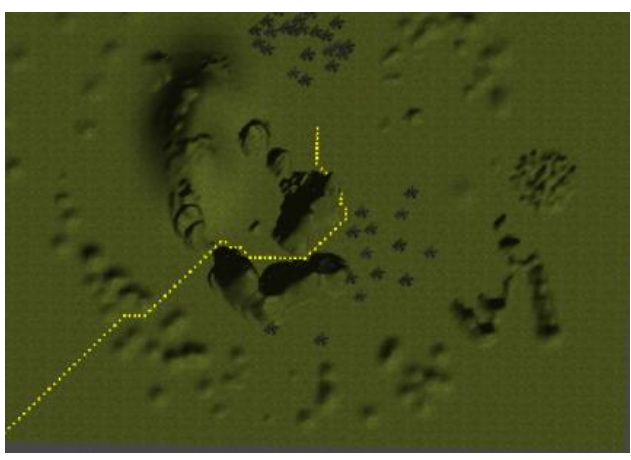

Figure 5. The effect picture of 2.5D path planning

Different route optimization algorithms are used on the map of 2.5-dimensional grid, as is shown in Table 3. Time and iteration is expressed using integer.

TABLE III. COMPARISON TABLE OF DIFFERENT ALGORITHMS

\begin{tabular}{|l|l|l|l|}
\hline Algorithm & $\begin{array}{l}\text { Path } \\
\text { length }\end{array}$ & Time & Iterations \\
\hline $\mathrm{A}^{*}$ & 16.9 & $8 \mathrm{~ms}$ & 245 \\
\hline IDA* & 19.24 & $1937 \mathrm{~ms}$ & 0 \\
\hline Dijkstra & 16.9 & $29 \mathrm{~ms}$ & 1047 \\
\hline Improved JPS & 16.9 & $3 \mathrm{~ms}$ & 24 \\
\hline
\end{tabular}

As is shown in Table 3, the improved JPS algorithm can improve the average speed about two times higher than the $\mathrm{A} *$ algorithm, reduce the number of iterations about 10 times, and the search space is greatly reduced. As for IDA * and Dijkstra algorithm, the speed is greatly improved, and the search space is reduced, which improves the efficiency of the algorithm largely.

\section{CONCLUSION}

The experimental environment: Asus F80C laptop, graphics card is ATI Mobility Radeon HD3470, CPU is Intel celeron $\mathrm{D}$, clocked at $1.2 \mathrm{GHZ}$.

In this study, fuzzy logic is used to evaluate the trafficability of the rough road. The trafficability factor is used in the improved JPS algorithm, so simulation is closer to reality. 


\section{REFERENCES}

[1] S.S. Ge, Y.J. Cui, "New potential functions for mobile robot path planning", IEEE Transaction on Robotics and Automation, vol.16, no.5, pp.615-620, 2000.

[2] S.A. Masoud, A.A, Masoud, "Motion Planning in the Presence of directional and regional avoidance constraints using nonlinear, anisotropic, harmonic potential fields: a physical metaphor", IEEE Transaction on System, Man, and Cybernetics, Part A, vol. 32, no.6, pp.705-723, 2002.

[3] Bao JIN, Yi-Li FU, Shu-guo WANG, Zheng-cai CAO, "Robot fuzzy motion planning approach in unknown environment. Journal of Harbin Institute of Technology", vol.37, no.10,pp.1315-1317, 2005.

[4] D. Harabor, A. Botea, P.Kilby, "Path Symmetries in Uniform-cost Grid Maps", In Proceedings of 9th Symposium on Abstraction Reformulation and Approximation, Barcelona, Spain, 2011.

[5] D. Harabor and A. Botea,"Breaking Path Symmetries in 4connected Grid Maps". In Proceedings of the 6th Annual AAAI Conference on AI and Interactive Digital Entertainment, Stanford University, Palo Alto, CA, USA, 2010.
[6] D. Harabor and A. Grastien, "Online Graph Pruning for Pathfinding on Grid Maps", In Proceedings of the 25th National Conference on Artificial Intelligence, San Francisco, USA., 2011.

[7] D. Harabor and A.Grastien,"The JPS Pathfinding System", In Proceedings of the 5th Symposium on Combinatorial Search, Niagara Falls, Canada, 2012.

[8] M.J. Smith, Geospatial Analysis-Principles of Technology and Software Tools, Beijing, Electronic Industry Press, 2009.

[9] Kangcong Zhang, Introduction of Geographic Information Systems, Beijing, Tsinghua University Press, 2009.

[10] E.H.L. Fong, W. Adams, F.L. Crabbe , A.C. Schultz, "Representing a 3D environment with a 21/2-D map structure", In IEEE/RSJ International Conference on Intelligent Robots and Systems, Las Vegas, pp.2986-2991, 2003.

[11] J.S. Gutmann, M. Fukuchi, M. Fujita,"A floor and obstacle height map for 3D navigation of humanoid robot",In IEEE International Conference on Robotics and Automation, Sendai, Japan, pp.10661071,2005 . 